\title{
CORRELATIVE EXPLORATION OF EGG SIGNALS FOR DIRECT BRAIN-COMPUTER COMMUNICATION
}

\author{
Gary N. Garcia, Touradj Ebrahimi, Jean-Marc Vesin. \\ Swiss Federal Institute of Technology - EPFL, CH-1015 Lausanne, Switzerland \\ Gary.garciadepfl.ch, Touradj.Ebrahimidepfl.ch, \\ Jean-Marc.Vesindepfl.ch
}

\begin{abstract}
In this study we present a method for classifying EEG signals based on the information content of their correlative time-frequency-space representation (CTFSR). A support vector machine (SVM) kernel is proposed that can be calculated in the time domain while it computes a similarity measure in the CTFSR space. This classification method is used in a brain-computer interface (BCI) application.

The use of the SVM approach allows us to propose a simple strategy for adapting the BCI to possible long term variations in the brain activity.
\end{abstract}

\section{INTRODUCTION}

An electroencephalogram (EEG) is the measurement of electrical potentials in the brain, by an array of electrodes placed at the scalp or on the brain's cortex. The relative simplicity, good time resolution and non-invasiveness of the scalp-measured EEG are the main reasons that made it a privileged tool for monitoring brain activity in therapeutic and neurophysiologic research. In the sequel, we use the term EEG for a scalp-measured EEG.

Since the first experiments, there is clear evidence that observable changes in EEG result from performing given mental activities [1]. Under the light of this evidence it becomes possible to conceive a communication system between human brain and a computer, in which the information support is the EEG pattern, voluntarily generated by the user and independent of any muscular activity. Such a system is called a Brain-Computer Interface (BCI) and is the object of intense research, mainly for people with severe motor disabilities [2]. "Think and make it happen without any movement" is a dream that might become reality through a BCI.

A BCI is composed of three subsystems, namely signal acquisition, signal processing and output generation. (Figure 1)

The acquisition subsystem is responsible for the measurement and digitization of EEG signals. These signals are often noisy and may contain artifacts (due to muscular and ocular movements) that can mask the EEG patterns generated by the user.

The signal processing subsystem is generally subdivided into a preprocessing unit, responsible for artifact detection, and a feature extraction and recognition unit that determines the command sent by the user to the BCI. This command is sent to the output subsystem, which generates a "system answer" that constitutes a feedback to the user who can modulate his mental activities so as to produce those EEG patterns that make the BCI accomplish his intents.

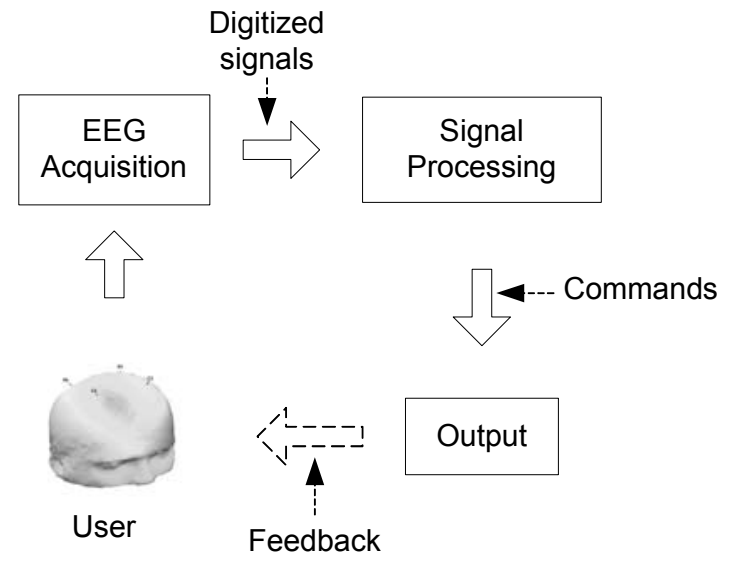

Figure 1. General BCI architecture.

Figure 2 illustrates the basic scheduling of a BCI. We define the BCI period as the average time between two consecutive system answers and the EEG trial duration as the amount of EEG (in terms of time) that the BCI needs to analyze in order to generate an answer. These parameters are crucial for the usability of the BCI and depend on the brain dynamics, the type of mental activities (MAs) used and the computation load.[2]

In this paper we focus on the recognition of EEG patterns (associated with given MAs) by the BCI and we propose a strategy for adapting the system to possible long term 
variations in the EEG patterns that can appear as a result of different brain's background activities over time.

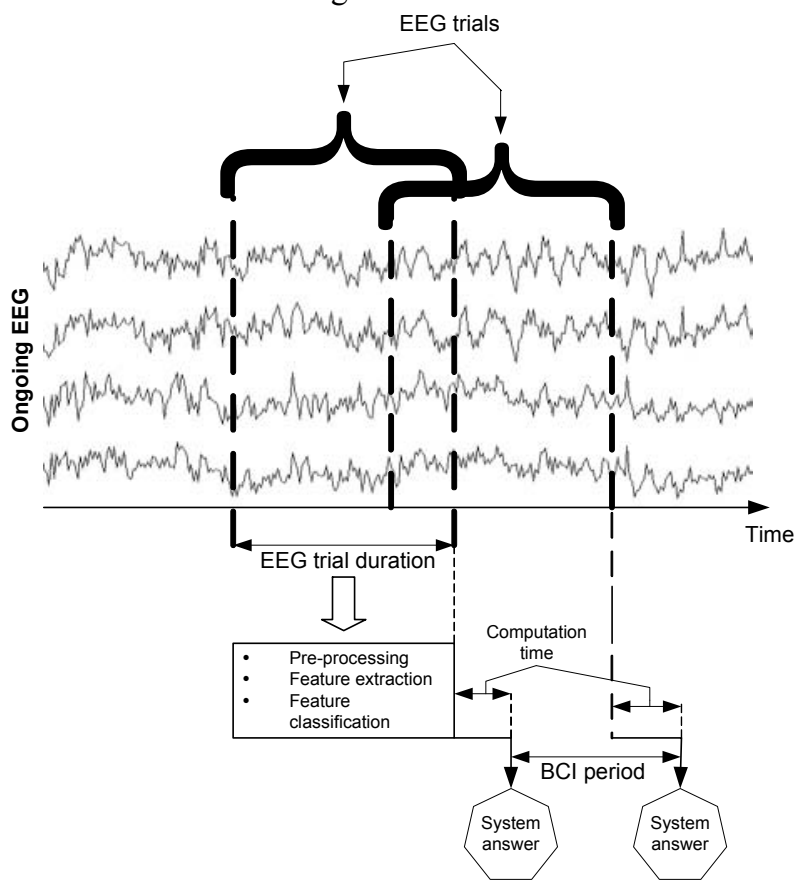

Figure 2. BCI scheduling.

\section{TRENDS IN EEG ANALYSIS FOR BCI APPLICATIONS}

In the context of BCI, EEG signals were mainly analyzed in the time, frequency, and time-frequency domains.

Most of the research groups work in the frequency domain and extract the information characterizing mental activities from the nonparametric and parametric spectral representations of EEG [2]. Also, the joint spectral properties of the EEG components are analyzed in [3] for detecting particular emotional states.

The relationship between the time courses of the signals coming from different electrodes serves as an indication of motor activities in [4]. Useful information can also be extracted from particular brain configurations that can be interpreted in terms of brain states [5][6].

Time-frequency and time-scale representations of EEG signals were exploited for finding those neuronal groups that synchronize their activity as a response to a particular stimulus (event related potentials) [7][8].

From the above considerations it can be stated that mental activities, when mapped onto the time-frequency representation of EEG signals, display a picture that illustrates the cooperative activity of neuronal groups. A possible way to explore this activity consists in analyzing the joint time-frequency-space correlations between the components of an EEG signal.

\section{TIME-FREQUENCY-SPACE CLASSIFICATION OF EEG TRIALS}

The BCI is first presented with a set of labeled EEG trials (training set) i.e. recorded during the performance of known MAs, in order to build the classifier.

Let the training set be $\Gamma=\left\{\left(X_{i}, y_{i}\right) ; 1 \leq i \leq L\right\}$ where $y_{i}$ is the label of the EEG trial $X_{i}$.

For our purposes we consider the two-class classification problem, i.e. $y_{i} \in\{-1,+1\}$. Multi-class classification can be done with multiple pairwise comparisons. The binary classification problem is solved here using the support vector machine (SVM) approach [9].

The goal is to determine a mapping $\Phi$, from the EEG trial space $\underline{\mathbf{X}}$ into a feature space $\underline{\mathbf{F}}$ (endowed with an inner product) such that the classes can be separated by a hyperplane in $\underline{\mathbf{F}}$.

Therefore, the decision function $f$ for an EEG trial $X \in \underline{\mathbf{X}}$ is $f: \underline{\mathbf{X}} \rightarrow\{-1,+1\}$

$$
X \rightarrow f(X)=\operatorname{sign}(\langle\Phi(X), w\rangle+b) ; w \in \underline{\mathbf{F}}, b \in \mathbb{R}
$$

The SVM theory has established that one does not need to explicitly calculate the mapping $\Phi(\bullet)$ if there is a kernel function $k(\bullet, \bullet)$ such that

$$
\langle\Phi(X), \Phi(\tilde{X})\rangle=k(X, \tilde{X})
$$

Some classical types of kernels satisfying the above property are: the polynomial, radial Gaussian and sigmoidal kernels [9].

In this study we propose to map $\underline{\mathbf{X}}$ on the space $\underline{\mathbf{F}}$, defined by the correlative time-frequency-space representations (CTFSR) of the elements in $\underline{\mathbf{X}}$. The CTFSR choice is motivated by the promising results obtained in previous works [10].

The CTFSR of an EEG trial $X(t)$, composed of the signals measured at $N$ electrodes, is an $N \times N$ matrix defined as.

$\Phi_{(X)}(\theta, \tau)=\left[\begin{array}{ccc}A_{x 1, x 1}(\theta, \tau) & \ldots & A_{x 1, x N}(\theta, \tau) \\ \ldots & \ldots & \ldots \\ A_{x N, x 1}(\theta, \tau) & \ldots & A_{x N, x N}(\theta, \tau)\end{array}\right]$

where $X(t)=\left[\begin{array}{lll}x_{1}(t) & \ldots & x_{N}(t)\end{array}\right]^{\mathrm{T}}, \quad(\cdot)^{\mathrm{T}}$ is the transpose operator, $\theta$ and $\tau$ are the frequency and time lags respectively, and $A_{x m, x n}(\theta, \tau)=\int x_{m}(t+\tau / 2) \cdot x_{n} *(t-\tau / 2) e^{j \theta t} d t$ is the cross correlative time-frequency function (also called ambiguity function [11]) between $x_{m}(t)$ and $x_{n}(t)$.

We define the kernel function between two EEG trials $X$ and $\tilde{X}$ as

$$
k(X, \tilde{X})=\mathbf{1}_{N} \cdot \iint \Phi_{(X)}(\theta, \tau) \cdot \Phi_{(\tilde{X})}^{\mathrm{H}}(\theta, \tau) d \theta d \tau \cdot \mathbf{1}_{N}^{\mathrm{T}}
$$


where $\mathbf{1}_{N}$ is a $1 \times 1$ real matrix with unit components, and $(\bullet)^{\mathrm{H}}$ is the Hermitian operator.

Substituting Eq. (2) into Eq. (3) we get. $k(X, \tilde{X})=\mathbf{1}_{N} \cdot \iint X(t+\tau) \cdot X^{\mathrm{H}}(t) \cdot \tilde{X}(t) \cdot \tilde{X}^{\mathrm{H}}(t+\tau) d t d \tau \cdot \mathbf{1}_{N}^{\mathrm{T}}$ The last result shows that we do not need to explicitly compute $\Phi_{(X)}$ and $\Phi_{(\tilde{X})}$ as only $k(X, \tilde{X})$ matters.

The estimates for $w \in \underline{\mathbf{F}}$ and $b \in \mathbb{R}$ are found by minimizing the regularized risk $R_{\text {reg }}$ which depends on the classifier complexity and the empirical risk $R_{e m p}$ :

$$
\begin{aligned}
& (w, b)=\underbrace{\arg \min }_{w \in \mathbf{E}, b \in \mathbb{R}}\left[\frac{1}{2}\langle w, w\rangle-v \rho+\mathrm{R}_{\mathrm{e} m p}\right] \\
& \mathrm{R}_{\mathrm{e} m p}=\frac{1}{L} \sum_{i=1}^{L} \max \left\{0, \rho-y_{i} \cdot f\left(X_{i}\right)\right\}
\end{aligned}
$$

The support vectors (SVs) are those training points for which $y_{i} \cdot f\left(X_{i}\right) \leq \rho, \rho$ is called the loss parameter, $v$ is a lower bound on the fraction of training points that are SVs [9].

The results in [9] show that $w$ is a linear combination of $\left\{y_{i} \cdot \Phi\left(X_{i}\right) ; 1 \leq i \leq L\right\}$

$$
w=\sum_{i=1}^{L} \alpha_{i} y_{i} \cdot \Phi\left(X_{i}\right)
$$

and that solving Eq. (4) is equivalent to find

$$
\max \left[-\frac{1}{2} \sum_{i_{1}, i_{2}=1}^{L} \alpha_{i_{1}} \alpha_{i_{2}} y_{i_{1}} y_{i_{2}} k\left(X_{i_{1}}, X_{i_{2}}\right)\right]
$$

subject to constraints

$$
\begin{aligned}
& \sum_{i=1}^{L} y_{i} \alpha_{i}=0 ; 0 \leq \alpha_{i} \leq \frac{1}{L} \\
& \sum_{i=1}^{L} \alpha_{i} \geq v
\end{aligned}
$$

The offset $\mathrm{b}$ and the loss parameter $\rho$ can be found using

$y_{i} \cdot\left(\left\langle w, \Phi\left(X_{i}\right)\right\rangle+b\right)=\rho \quad$ when $\alpha_{i} \in\left(0, \frac{1}{L}\right)$

A key requirement in BCI applications consists in the regular adaptation of the classifier to the possible changes in user's mental activities [2]. As only the SVs determine the classification parameters ( $w$ and $b$ ) we can easily update them by including the old SVs in a new training set.

\section{BCI IMPLEMENTATION}

Three types of MAs were used: imagination of left (MA1) or right (MA2) index finger movements and a baseline (MA3) where the subject can imagine anything except MA1 or MA2. The goal was to allow the user to control the movement, to the left or to the right, of a cursor on a computer screen.

Our BCI implementation can be explained in terms of three states: the neutral state in which the BCI whether recognizes MA3 or cannot recognize any known MA, the active state in which the BCI recognizes MA1 or MA2 and answers with an action and a transition state between the above mentioned states.

State changes occur at a rate defined by the BCI period, and are determined by the activation of two Boolean variables: detection (B1) and confirmation (B2). B1 is true when MA1 or MA2 are recognized and $\mathrm{B} 2$ is true when $\mathrm{B} 1$ is true and if the $M$ previously recognized MAs are equal to the currently recognized MA (Figure 3). The parameter $M$ (latency time) depend on the rate of false positive recognitions, although it would not be larger than two seconds.

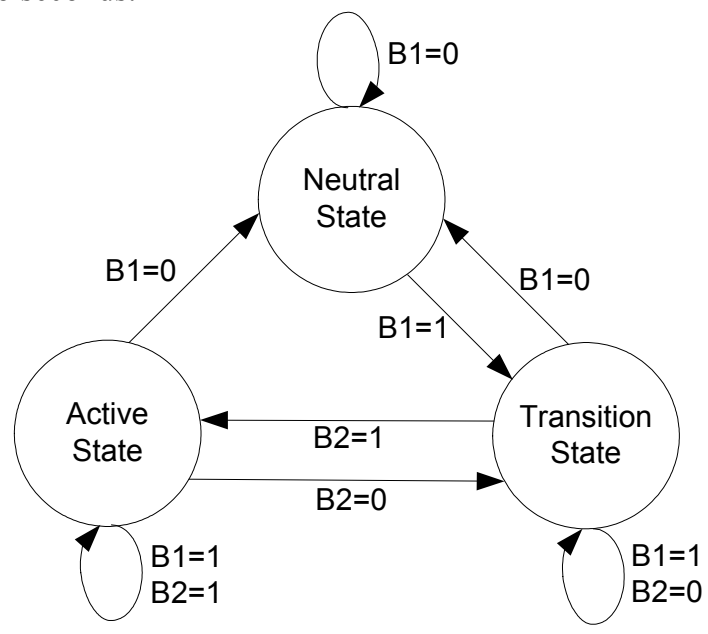

Figure 3. BCI states.

The set of BCI parameters are summarized in Table 1.

\begin{tabular}{|l|}
\hline BCI period \\
\hline EEG trial duration \\
\hline Classification parameters \\
\hline Latency time \\
\hline
\end{tabular}

Table 1. BCI parameters.

The optimal values for these parameters are determined during several training sessions and continuously updated.

\section{RESULTS AND DISCUSSIONS}

Two male right-handed subjects participated in six twenty-minute sessions. The signals from electrodes: Fp1, Fp2, C1, C3, C4, C2, P3 and P4 of the 10-20 International System [1] were measured.

The labeled EEG trials recorded in the first training session were used for estimating a preliminary set of classification parameters. In the next training sessions 
continuous feedback was provided to the subjects, indicating if the MA they were requested to perform was successfully recognized or not. The feedback of a session was provided using the updated classification parameters of the precedent session.

The first five sessions were devoted to training. In the last session, the subjects were asked to move the cursor in a 2D maze so as to reach one of the exit doors.

Each session was preceded by a short calibration period in which the BCI was adjusted to the subject. In the meantime, the subject could see a representation of his EEG signals mapped onto a $2 \mathrm{D}$ or $3 \mathrm{D}$ scene in order to get familiarized with the system.

After the first training session, the BCI period and the EEG trial duration were chosen among three possible alternatives depending on the classification error (Table 2). According to these results the BCI period and the EEG trial duration were set to 250 and 500 milliseconds respectively.

\begin{tabular}{|l|c|c|c|}
\hline & $\mathbf{0 . 1 2 s} / \mathbf{0 . 2 5} \mathrm{s}$ & $\mathbf{0 . 2 5 s} / \mathbf{0 . 5 s}$ & $\mathbf{0 . 5 s} / \mathbf{1 s}$ \\
\hline Subject 1 & $52 \%$ & $42 \%$ & $46 \%$ \\
\hline Subject 2 & $48 \%$ & $38 \%$ & $45 \%$ \\
\hline
\end{tabular}

Table 2. Classification error rate in the first training session for different values of BCI period/EEG trial duration

In Figure 4, we report the true and false positives rates of the last four sessions of training. As it can be observed the true positives rate increased over the sessions for both subjects reaching $86 \%$ for subject 2 .

The false positive rate is not larger than $33 \%$ for subject 1 and $30 \%$ for subject 2 . As this result is not good enough we set the latency time to one BCI period (i.e. 250 milliseconds), so that the system waits a confirmation of one EEG trial before executing any action. This parameter can reach the suitable value of zero if the user is able to decrease his false positives rate below $10 \%$. Thus, we can reward the user if he improves his ability to use the BCI.

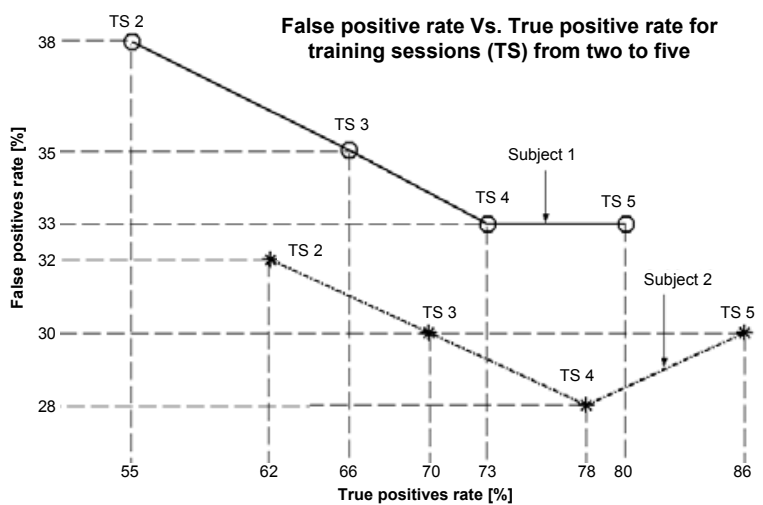

Figure 4. Evolution of the false positives vs. true positives rate for both subjects.

\section{CONCLUSIONS}

In this paper we presented a method for classifying EEG trials, based on the information content of their time-frequency-space representation. The dimensionality of this representation that makes it difficult to manipulate [10] was avoided using SVM concepts and a kernel that can be calculated in the time domain. We also proposed a method for easy updating of the classifier parameters by adding the old support vectors to the next training set.

Finally, we introduced a BCI implementation that can be adapted to the user performance and brain dynamics.

As the immediate goal of our research is to provide control of a cursor in a $2 \mathrm{D}$ environment, we need to explore the recognition of at least five MAs. Since part of the success of a BCI depends on the user himself the feedback strategy needs to be carefully designed by taking into account physiological and psychological aspects.

\section{REFERENCES}

[1] A. Gevins, "The future of electroencephalography in assessing neurocognitive functioning," EEG. and Clin. Neurophysiol., vol. 106, pp. 165-172, 1998.

[2] J.R. Wolpaw, et al, "Brain-computer interfaces for communication and control," Clin. Neurophysiol., vol. 113, pp. 767-791, 2002.

[3] A. Choppin, "Emotion Expression with Neural Networks," M. Sc. Thesis Dept. Inf. Proc. Tokyo Institute of Technology, Japan, 2000.

[4] A.B. Barreto, et al, "Spatio-temporal EEG patterns associated with voluntary motion preparation," IEEE Int. Conf. Eng. in Med. and Biol. Soc., vol. 2, pp. 857-858, 1995.

[5] E. Haselsteiner and G. Pfurtscheller, "Using TimeDependent Neural Networks for EEG Classification," IEEE. Trans. Rehab. Eng., vol. 8, no. 4, pp. 457-463, Dec. 2000.

[6] B. Obermaier, et al, "Hidden Markov models for online classification of single trial EEG data," Pattern Recog. Lett., 22, pp. 1299-1309, 2001.

[7] Chang Su Ryu, et al, "A Time-Frequency Analysis of the EEG Evoked by Negative and Positive Visual Stimuli," IEEE Int. Conf. Eng. in Med. and Biol. Soc., vol. 20, no. 4 , pp. 2012-2015, 1998.

[8] P.J. Durka, et al, "Time-Frequency Analysis of Stimulus-Driven EEG Activity by Matching Pursuit," IEEE Int. Conf. Eng. in Med. and Biol. Soc., pp. 1009-1010, 1996.

[9] B. Schölkopf and A. Smola, Learning with Kernels, MIT Press, 2002.

[10] G. Garcia, T. Ebrahimi, J-M. Vesin, "Classification of EEG signals in the ambiguity domain for brain computer interface applications," IEEE Int. Conf. Dig. Signal Proc., vol. 1, pp. 301-305, 2002.

[11] M.G. Amin, et al, "The Spatial Ambiguity Function and Its Applications," IEEE Sig. Proc. Lett., vol. 7, no. 6, pp. 138-140, June 2000. 